\title{
COMBINED USE OF METFORMIN AND PIOGLITAZONE THAN METFORMIN ALONE IN GLUCOSE INTOLERANCE PATIENTS WITH NON- ALCOHOLIC FATTY LIVER DISEASE SHOWED SIGNIFICANT RADIOLOGICAL AND BIOCHEMICAL IMPROVEMENT
}

\author{
AMIN MF ${ }^{1}$, SULTANA SR ${ }^{2}$, PRASAD I ${ }^{3}$, RAHIM MA ${ }^{4}$, RAHMAN MA ${ }^{5}$, MIR ABMRK ${ }^{6}$
}

\begin{abstract}
:
Context: Non Alcoholic Fatty liver disease (NAFLD) is a hepatic manifestation of metabolic syndrome. The pathogenesis of steatosis and cellular injury is thought to be related mostly to insulin resistance. Insulin sensitizing drugs showed promising results in number of trials. This was an open label clinical trial in newly detected glucose intolerant patients with NAFLD, to evaluate the effectiveness and superiority of pioglitazone and metformin combination to metformin alone.
\end{abstract}

Methods: Forty nine patients with newly detected abnormal glucose tolerance, naïve to any anti diabetic drug, were randomly selected, from the gastroenterology out-patient department of BIRDEM Hospital, Dhaka, with the findings of ultasonographic changes of fatty liver and raised ALT and assigned to 6 months treatment with pioglitazone $30 \mathrm{mg}$ plus metformin 1700 mg daily (Group 1, $n=27$ ) or only metformin $1700 \mathrm{mg}$ alone (Group 2, $n=22$ ).

Results: Mean age of the study population was $45.80 \pm 8.54$ years, Male female distribution of the study subjects were $65.3 \%$ and $34.7 \%$ respectively. Significant reduction of ALT, F, ABF, HbA1c, cholesterol, triglyceride of the study population were achieved either by metformin

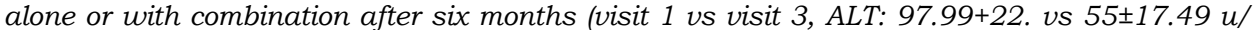
l; Fasting sugar: $9.1+1.9$ vs6.64 $\pm 0.94, \mathrm{mmol} / \mathrm{l}$; $A B F$ : $13.3 \pm 2.5$ vs $8.69 \pm 1.21 \mathrm{mmol} / \mathrm{l}$; HBA1c: $8.1 \pm 0.9$ vs $6.87 \pm 0.57 \%$; Cholesterol: $205.9 \pm 30.1$ vs $186.12 \pm 22.26 \mathrm{mg} / \mathrm{dl}$; $\mathrm{TG}$ : $230.4+48.1$ vs 166.2 \pm 31.82$)$. In comparison between two groups, Group 1 had found to be significantly better glycemic control compared to their counterpart at the end of 6 months (Group 1 vs Group 2,

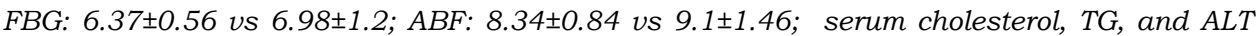
levels were also found to be significant change as Cholesterol: $178.89 \pm 18.59$ vs $195 \pm 23.55$ $\mathrm{mg} / \mathrm{dl}$; TG: $155.85 \pm 20.99$ vs $178.91 \pm 38.24 \mathrm{mg} / \mathrm{dl} ; \mathrm{ALT}$ : $55 \pm 17.49$ vs $45.74 \pm 12.63 \mathrm{u} / \mathrm{L}$. In final visit, ultrasonographic change also found to be significantlyimproved from fatty change to normal in patients with both metformin and pioglitazone group than patients on metformin alone.

Conclusion: Treatment of NAFLD of newly detected Type 2 DM or IGT patients with high ALT by both metformin and pioglitazone is more effective in reduction of ALT and lipids and also able to reverse the severity of fatty changes of liver towards normal significantly.

Key words: Alanine aminotransferase, diabetes mellitus, impaired glucose tolerance, nonalcoholic fatty liver disease.

J Dhaka Med Coll. 2013; 22(2) : 188-194.

1. Dr. Mohammod Feroz Amin, Assistant Professor, Department of Endocrinology, Ibrahim Medical College and BIRDEM General Hospital, Dhaka.

2. Dr. Syeda Rezina Sultana, Senior Medical Officer, OPD, BIRDEM, Dhaka.

3. Dr. Indrajit Prasad, Assistant Professor, Department of Endocrinology, Dhaka Medical College, Dhaka.

4. Dr. Muhammad Abdur Rahim, Registrar, Department of Internal Medicine, Ibrahim Medical College and BIRDEM General Hospital, Dhaka.

5. Prof. Md. Anisur Rahman, Professor, Department of GastroIntestinal, Hepatobiliary and Pancreatic Diseases, Ibrahim Medical College and BIRDEM General Hospital, Dhaka.

6. Dr. ABM Rezaul Karim Mir, Lecturer, Dept. of Pharmacology, Dhaka Medical College, Dhaka

For correspondence: Dr. Mohammod Feroz Amin, Assistant Professor, Department of Endocrinology, Ibrahim Medical College and BIRDEM General Hospital, Dhaka. Cell Phone: +8801715010102, E-mail: feroz_amin@yahoo.com 


\section{Introduction}

NAFLD is the hepatic manifestation of metabolic syndrome. In fact, risk factors associated with NAFLD include central obesity, type-2 diabetes mellitus, insulin resistance (IR) and dyslipidemia. ${ }^{1}$ Non-alcoholic fatty liver disease (NAFLD), is becoming recognized as a condition possibly involved in the pathogenesis of these diseases. Support for this hypothesis emerges from studies revealing that NAFLD precedes the manifestations of the metabolic derangements. ${ }^{2,3}$ The spectrum of NAFLD may be from simple hepatic steatosis to nonalcoholic steatohepatitis (NASH) which may be complicated to cirrhosis and even hepatocellular carcinoma. ${ }^{4,5}$ The term NAFLD is used to describe a condition of fat accumulation in liver in absence of excessive alcohol consumption (<20 g/day) and other specific causes of hepatic steatosis. NAFLD affects some $10-24 \%$ of general population and the prevalence increases upto 6 foldin obese persons. ${ }^{6}$ NAFLD is the cause of asymptomatic elevation of ALT in up to $90 \%$ of cases. ${ }^{7}$ Regardless of body mass index (BMI), the presence of type $2 \mathrm{DM}$ significantly increases the risk and severity of NAFLD. ${ }^{4,5}$ Trunkal obesity is an important risk factor even in a patients with a normal BMI. ${ }^{8}$ About half the patients with hyperlipidemia are found to have NAFLD on ultrasonography. ${ }^{9}$ Most of the patients with NAFLD are asymptomatic at diagnosis. Common symptoms are fatigue, malaise, right upper quadrant pain or discomfort and sensation of fullness. Hepatomegaly is common. ${ }^{10,11}$ Other findings relate to obesity and other features of metabolic syndrome. Mild to moderate increased serum levels of aspartate aminotransferese (AST), ALT or both are the most common and often the only laboratory abnormality. The ratio of AST: ALT is usually $<1$ and the ratio increases as fibrosis advances. ${ }^{10}$ Hypertriglyceridemia increases the risk of NAFLD. ${ }^{9}$ Ultrasonography is the most widely used, allows detecting moderate and sever steatosis with a fair sensitivity and specificity only when fat on liver biopsy exceeds $33 \%{ }^{10}$. The American Association for study of liver disease (AASLD) set the limit for diagnosis of NAFLD at fat accumulation in the liver of at least 5 to $10 \%$. NAFLD is estimated as the percentage of fat leaden hepatocytes observed by light microscopy in liver biopsy ${ }^{10}$. On USG fatty infiltration of the liver produces a diffuse increase in echogenecity as compared with that of the kidneys. USG has a sensitivity of $89 \%$ and specificity of $93 \%$ in detecting steatosis and sensitivity and specificity of $77 \%$ and $89 \%$ respectively, in detecting increased fibrosis ${ }^{11}$. Good metabolic control in DM or dyslipidaemia is not always effective in reversing NAFLD. Weight loss shows improvement on liver test results. ${ }^{12-15}$ No medications have been proved to directly reduce or reverse liver damage independent of weight loss. Many drugs have been used with variable degree of improvements in liver function tests and hepatic histology. ${ }^{16-21}$ Emerging evidence confirms that NAFLD can be progressive and associated with significant morbidity and mortality. Despite efforts aimed at improving early detection and prevention, many patients are still seen at the advanced stages. ALT is the best and most reliable noninvasive method for screening NAFLD. Over the last few years, clinical trials evaluated the use insulin sensitizers in treatment of NAFLD. Mixed results, heterogeneous therapeutic approaches and the small numbers of subjects have limited their applications as clinical guideline. In the current study, we have tried to evaluate the effectiveness of the combined insulin sensitizers which would act on different receptors at the tissue level.

\section{Methods}

This open level clinical trial was carried out in the outpatient (OPD) wing of Department of Gastrointestinal, Hepatobiliary and Pancreatic Diseases (GHPD) at Bangladesh Institute of Research and Rehabilitation in Diabetes, Endocrine and Metabolic Disorders (BIRDEM), Dhaka, in the year 2010. Sixty newly detected DM and IGT patients, referred at GHPD-OPD for raised ALT and ultrasound proven NAFLD were enrolled in this study. Patients with chronic liver disease (CLD), positive HBsAg and anti-HCV and history of alcoholism were excluded from the study. All patients were on hypocaloric diet. Every alternate patients were prescribed either 
metformin $850 \mathrm{mg}$ twice daily and pioglitazone $30 \mathrm{mg}$ once daily (Group 1) or metformin 850 mg twice daily (Group 2) for six months. Total 49 patients (27 patients in group 1 and 22 in group 2) completed the trial. Patients were assessed clinically and by laboratory investigations at enrolment (visit 1), at $3^{\text {rd }}$ (visit 2 ) and at $6^{\text {th }}$ months (visit 3). Satistical analysis was done by using frequencies and percentages and by the applying paired sample test within the group and unpaired t test and chi-square test between groups and $p$ value of $<0.05$ was considered significant. Ethical clearance was obtained from ethical review committee of BIRDEM before starting the trial and informed written consent was taken from all patients before enrolment.

\section{Results}

Total number of patients was 49. Mean age was $45.80 \pm 8.54$ years. Male were $65.3 \%$ and female were $34.7 \%$. Baseline and anthropometric measurements of study population were shown in Table 1 . Thirteen $(26.5 \%)$ patients were asymptomatic, $14(28.6 \%)$ patients had a feeling of abdominal fullness, 10 (20.4\%) patients complained about upper abdominal pain and 12 $(24.5 \%)$ complained about both upper abdominal pain and fullness. Twenty seven (55.1\%) patients were treated by metformin $850 \mathrm{mg}$ twice and pioglitazone $30 \mathrm{mg}$ once daily (Group 1) and 22 $(44.9 \%)$ patients were treated by metformin 850 mg twice daily (Group 2). Significant improvements were noted with treatment in metabolic control like fasting and post prandial blood glucose, HbA1c and lipid profile in both groups in $2^{\text {nd }}$ and $3^{\text {rd }}$ visits (Table-II $\&$ III). Values of ALT showed significant improvements in $2^{\text {nd }}$ and $3^{\text {rd }}$ visits (Table-IV). The inter-group difference regarding ALT, FBG and TG were significant in every visit (Table-V). Though the inter-group difference regarding $\mathrm{ABF}$, total cholesterol and $\mathrm{HbA} 1 \mathrm{c}$ was not significant at $1^{\text {st }}$ visit, it became significant in subsequent visits (Table-V). At baseline, all patients were grade 1 fatty change or more in USG but in final visit significant improvement towards normal and more in the group treated with both metformin and pioglitazone (Table-VI).

Table-I

Baseline characteristics and anthropometric measurements of study population

\begin{tabular}{lcc}
\hline Variables & $\begin{array}{c}\text { Group 1 } \\
(\mathrm{n}=27)\end{array}$ & $\begin{array}{c}\text { Group 2 } \\
(\mathrm{n}=22)\end{array}$ \\
\hline Age (years) & $46.0 \pm 9.3$ & $45.4 \pm 5.7$ \\
Sex (male, female) $(\%)$ & $74.1,25.9$ & $54.5,45.5$ \\
DM, IGT (\%) & $92.6,7.4$ & $81.8,18.2$ \\
Height (cm) & $159.9 \pm 6.1$ & $157.6 \pm 7.9$ \\
Weight (kg) & $69.4 \pm 8.5$ & $68.5 \pm 11.0$ \\
BMI (kg/m2) & $27.1 \pm 2.2$ & $27.4 \pm 3.2$ \\
Waist circumference (cm) & $90.6 \pm 4.2$ & $91.3 \pm 4.7$ \\
Hip (cm) & $93.5 \pm 3.5$ & $94.1 \pm 4.1$ \\
\hline
\end{tabular}

Table-II

Biochemical parameters of group 1 in three visits

\begin{tabular}{lcccc}
\hline Variables & Visit & mean \pm SD & Comparison between visits & P value \\
\hline & 1 & $8.89 \pm 1.44$ & V1-V2 & $<0.001$ \\
FBG $(\mathrm{mmol} / \mathrm{L})$ & 2 & $7.04 \pm 0.79$ & $\mathrm{~V} 2-\mathrm{V} 3$ & $<0.001$ \\
& 3 & $6.37 \pm 0.50$ & $\mathrm{~V} 1-\mathrm{V} 3$ & $<0.001$ \\
& 1 & $13.20 \pm 2.07$ & $\mathrm{~V} 1-\mathrm{V} 2$ & $<0.001$ \\
$\mathrm{ABF}(\mathrm{mmol} / \mathrm{L})$ & 2 & $9.48 \pm 0.99$ & $\mathrm{~V} 2-\mathrm{V} 3$ & $<0.001$ \\
& 3 & $8.38 \pm 0.84$ & $\mathrm{~V} 1-\mathrm{V} 3$ & $<0.001$ \\
& 1 & $8.15 \pm 0.87$ & $\mathrm{~V} 1-\mathrm{V} 2$ & $<0.001$ \\
$\mathrm{HbA} 1 \mathrm{c}(\%)$ & 2 & $7.20 \pm 0.47$ & $\mathrm{~V} 2-\mathrm{V} 3$ & $<0.001$ \\
& 3 & $6.70 \pm 0.40$ & $\mathrm{~V} 1-\mathrm{V} 3$ & $<0.001$ \\
& 1 & $205.26 \pm 30.74$ & $\mathrm{~V} 1-\mathrm{V} 2$ & $<0.001$ \\
$\mathrm{CHOL}(\mathrm{mg} / \mathrm{dl})$ & 2 & $187.44 \pm 21.58$ & $\mathrm{~V} 2-\mathrm{V} 3$ & $<0.001$ \\
& 3 & $178.89 \pm 18.59$ & $\mathrm{~V} 1-\mathrm{V} 3$ & $<0.001$ \\
& 1 & $226.15 \pm 50.06$ & $\mathrm{~V} 1-\mathrm{V} 2$ & 0.004 \\
TG $(\mathrm{mg} / \mathrm{dl})$ & 2 & $177.26 \pm 26.62$ & $\mathrm{~V} 2-\mathrm{V} 3$ & $<0.001$ \\
& 3 & $155.85 \pm 20.99$ & $\mathrm{~V} 1-\mathrm{V} 3$ & \\
\hline
\end{tabular}

Data were expressed as maen $\pm \mathrm{SD}$. Mean values between the visits were compared by paired $\mathrm{t}$-test. 
Table-III

Biochemical parameters of group 2 in three visits

\begin{tabular}{|c|c|c|c|c|}
\hline Variables & Visit & Mean \pm SD & $\begin{array}{l}\text { Comparison } \\
\text { between visits }\end{array}$ & $\mathrm{P}$ value \\
\hline & 1 & $9.39 \pm 2.26$ & V1-V2 & $<0.001$ \\
\hline \multirow[t]{3}{*}{ FBG $(\mathrm{mmol} / \mathrm{L})$} & 2 & $7.88 \pm 1.53$ & V2-V3 & 0.003 \\
\hline & 3 & $6.98 \pm 1.20$ & V1-V3 & $<0.001$ \\
\hline & 1 & $13.39 \pm 2.93$ & V1-V2 & $<0.001$ \\
\hline \multirow[t]{3}{*}{$\mathrm{ABF}(\mathrm{mmol} / \mathrm{L})$} & 2 & $9.90 \pm 1.76$ & V2-V3 & $<0.001$ \\
\hline & 3 & $9.13 \pm 1.46$ & V1-V3 & $<0.001$ \\
\hline & 1 & $8.10 \pm 0.92$ & V1-V2 & $<0.001$ \\
\hline \multirow[t]{3}{*}{ HbA1c (\%) } & 2 & $7.34 \pm 0.76$ & V2-V3 & 0.003 \\
\hline & 3 & $7.03 \pm 0.71$ & V1-V3 & $<0.001$ \\
\hline & 1 & $206.55 \pm 29.90$ & V1-V2 & $<0.001$ \\
\hline \multirow[t]{3}{*}{$\mathrm{CHOL}$ (mg/dl) } & 2 & $191.14 \pm 26.12$ & V2-V3 & 0.291 \\
\hline & 3 & $195.00 \pm 23.55$ & V1-V3 & 0.034 \\
\hline & 1 & $235.59 \pm 46.22$ & V1-V2 & $<0.001$ \\
\hline \multirow[t]{2}{*}{ TG (mg/dl) } & 2 & $199.82 \pm 40.73$ & V2-V3 & 0.004 \\
\hline & 3 & $178.91 \pm 38.24$ & V1-V3 & $<0.001$ \\
\hline
\end{tabular}

Data were expressed as maen $\pm \mathrm{SD}$. Mean values between the visits were compared by paired ttest.

Table IV

Serum ALT levels in three visits in two groups

\begin{tabular}{ccccc}
\hline Group & Visit & ALT (U/L) & $\begin{array}{c}\text { Comparison } \\
\text { between visits }\end{array}$ & P value \\
\hline \multirow{2}{*}{1} & 1 & $91.52 \pm 23.14$ & V1-V2 & $<0.001$ \\
& 2 & $62.78 \pm 14.79$ & V2-V3 & $<0.001$ \\
& 3 & $45.74 \pm 12.63$ & V1-V3 & $<0.001$ \\
2 & 1 & $105.59 \pm 18.63$ & V1-V2 & $<0.001$ \\
& 2 & $81.77 \pm 16.29$ & V2-V3 & $<0.001$ \\
& 3 & $66.36 \pm 16.02$ & V1-V3 & $<0.001$ \\
\hline
\end{tabular}

Data were expressed as mean \pm SD. Mean values between visits were compared by paired ttest. 
Table-V

Significance of different biochemical parameters between two groups

\begin{tabular}{lcccc}
\hline Variables & Visit & Group & Mean \pm SD & P value \\
\hline \multirow{2}{*}{ FBG (mmol/L) } & 1 & 12 & $8.87 \pm 1.449 .39 \pm 2.26$ & 0.338 \\
& 2 & 12 & $7.04 \pm 0.797 .87 \pm 1.52$ & 0.018 \\
& 3 & 12 & $6.37 \pm 0.556 .98 \pm 1.20$ & 0.024 \\
ABF (mmol/L) & 1 & 12 & $13.20 \pm 2.0713 .38 \pm 2.93$ & 0.805 \\
& 2 & 12 & $9.48 \pm 0.999 .90 \pm 1.75$ & 0.299 \\
& 1 & 12 & $8.34 \pm 0.849 .13 \pm 1.46$ & 0.022 \\
HbA1c (\%) & 2 & 12 & $8.15 \pm 0.848 .11 \pm 0.92$ & 0.844 \\
& 3 & 12 & $7.21 \pm 0.477 .34 \pm 0.76$ & 0.459 \\
& 1 & 12 & $6.74 \pm 0.407 .03 \pm 0.71$ & 0.086 \\
CHOL (mg/dl) & 2 & 12 & $205.26 \pm 30.74206 .55 \pm 29.30$ & 0.883 \\
& 3 & 12 & $187.44 \pm 21.58191 .14 \pm 26.12$ & 0.590 \\
& 1 & 12 & $178.89 \pm 18.59195 .00 \pm 23.55$ & 0.010 \\
TG (mg/dl) & 2 & 12 & $226.15 \pm 50.06235 .59 \pm 46.22$ & 0.500 \\
& 3 & 12 & $177.26 \pm 26.62199 .82 \pm 40.73$ & 0.024 \\
& 1 & 12 & $155.85 \pm 20.99178 .91 \pm 38.24$ & 0.010 \\
ALT (U/L) & 2 & 12 & $91.52 \pm 23.14105 .59 \pm 18.63$ & 0.026 \\
& 3 & 12 & $62.78 \pm 15.7981 .72 \pm 16.29$ & $<0.001$ \\
\hline
\end{tabular}

Data were expressed as mean \pm SD. Maen values of two groups were compared by unpaired t-test.

Table-VI

USG report at final visit

\begin{tabular}{ccc}
\hline Normal USG in final visit & Treatment group & P value \\
\hline Metformin only & $4(16 \%)$ & 0.000 \\
Both metformin and pioglitazone & $21(84 \%)$ & \\
\hline
\end{tabular}

Value expressed as percentage $\%$ and $n=$ (number of the patients)

\section{Discussion}

Treatment strategies for NAFLD are improving the insulin resistance by weight loss, exercise and pharmacotherapy with insulin sensitizers. In our study, we have tried to evaluate and compare the efficacy of metformin and pioglitazone combination versus metformin alone for the reduction of ALT in NAFLD. Mean age of the study population was over 45 years. In a study $65.4 \%$ patients were in $40-59$ years age group. ${ }^{3}$ As previously noted, NAFLD is the hepatic manifestation of metabolic syndrome.
Risk factors associated with NAFLD include central obesity, type $2 \mathrm{DM}$, insulin resistance and dyslipidemia. ${ }^{4,23}$ The values of mean BMI, waist circumference, and TG in our patients clearly indicate that insulin resistance was risk factor for developing NAFLD in our population as well. In a population based study, it was found that waist circumference was recognized as a simple parameter for estimation of liver fat accumulation. It was found that presence of NAFLD correlates significantly with BMI and waist hip ratio which 
support our findings. ${ }^{6}$ Over the period of 3 and 6 months follow up, both the treatment groups showed improvements in $\mathrm{HbA} 1 \mathrm{c}$, more in combination group which confirmed the well established issues of glucose lowering effect of metformin and pioglitazone and more powerful blood glucose lowering effect of combination of both the drugs as stated in American Diabetic Association (ADA) guideline 2012 and guideline of American College of Clinical Endocrinology (AACE) 2012. A reduction in ALT is an acceptable end point for a proof of concept of exploratory trial in NAFLD. In our trial, serum ALT showed significant improvement at $3^{\text {rd }}$ and $6^{\text {th }}$ months in both groups. These finding confirmed the beneficial effect of insulin sensitizers in NAFLD as seen in other studies. ${ }^{18-22}$ These studies evaluated either metformin or thiazolidinediones versus diet and lifestyle measures and no study evaluated combination of insulin sensitizers. Our findings are in favor of combination of drugs. Abdominal ultrasonography could provide accurate information about hepatic steatosis, visceral obesity and metabolic syndrome in appearently healthy people who do not consume alcohol. ${ }^{11}$ In our study population no patients were found to normal USG finding in baseline, but in final visit $51 \%$ patients showed normal USG with no fatty change, $84 \%$ patient were in both metformin and pioglitazone drug.

Metformin is less expensive than most other treatment modalities of NAFLD. Most studies with prolonged use of metformin have shown no or little side effects and thus can be used safely for long period of time. For pioglitazone current recommendations are that the patients should be clinically monitored and it should not be used in those with advanced congestive cardiac failure (NYHA class III and IV).

\section{Conclusion}

In conclusion, NAFLD patients treated with metformin alone and combination of metformin and pioglitazone are effective in reducing ALT and also can reverse the ultrasonographic grading of fatty change towards normal and combination is better. Not only that, the combination group had better results in improving lipid profile and glycaemic status.
However, larger, multicenter studies can be done for better and more reliable results. However, our study had some limitations. Limited numbers of patients were evaluated for a short time. Liver biopsy was not done and patients were not followed up with fibroscan or ultrasonography.

\section{References}

1. Norbert S, Konstantinos K, Hans H. Causes of metabolic consequences of fatty liver. Endocrine Rev 2010; 29(7): 939-60.

2. Shibata M, KiharaY, Taguchi M, Tashiro M, Otsuki M. Non-alcoholic Fatty liver disease is a risk factor for type 2 diabetes in middle aged Japanese men. Diabetes Care 2007; 30: 2940-4.

3. Targher G, Bertolini L, Poli F, et al. Prevelance of non-alcoholic fatty liver disease and risk of future cardiovascular events among type 2 diabetic patients. Diabetes Care 2007; 54: 3541-6.

4. Silverman JF, O'Berin KF, Long S, et al. Liver pathology in morbidly obese patients with and without diabetes. Am J Gastroenterol 1990; 85: 1349-55.

5. Wanless IR, Lentz JS. Fatty liver hepatitis (steatohepatitis) and obesity: an autopsy study with analysis of risk factors. Hepatology 1990; 12: 1106-10.

6. Nomura H, Kashiwagi S,Hayashi J, et al. Prevalence of fatty liver in a general population of Okinawa, Japan. Jpn J Med 1998; 27: 142-9.

7. Daniel S, Ben Menachem T, Vasudevan G, Blumenkchl M. Prospective evaluation of unexplained chronic liver transaminase abnormalities in asymptomatic and symptomatic patients. Am J Gastroenterol 1999; 94: 3010-4.

8. Ruderman N, Chisholm D, Pi-Sunyer X, Schncider S. The metablically obese, normal weight individual revisited. Diabetes 1998; 47: 699-713.

9. Assy N, Kaita K, Mymin D, Levy C, Rosser B, Minuk G. Fatty infiltration of liver in hyperlipidemic patients. Dig Dis Sci 2000; 45: 1929-34.

10. Angulo P, Lindor KD. Non-alcoholic fatty liver disease. J GastroenterolHepatol 2002; 17 : 186-90.

11. Wieckowsaka A, McCullough AJ, Feldstien AE. Noninvasive diagnosis and monitoring of nonalcoholic steatohepatitis: present and future. Hepatology 2007; 46: 582-9.

12. LuyckxFh, Deasive C, Thirty A, et al. Liver abnormalities in severely obese subjects: effect of 
drastic weight loss in gastroplasty. Int $\mathrm{J}$ Obsess Relat Metab Disord 1998; 22: 222-6.

13. Anderson T, Gluud C, Franzmann MB, Cristoffersen P. Hepatic effects of dietary weight loss in morbidly obese subjects. J Hepatol 1991; 12: 224-9.

14. Palmr M, Schaffner F. Effect of weight reduction on hepatic abnormalities in overweight patients. Gastroenterology 1990; 99: 1408-13.

15. Uneo T, Sugawara H, Sujaku K, et al. Therapeutic effects of restricted diet and exercise in obese patient with fatty liver. J Hepatol 1997; 27 : 103-7.

16. Basanough M, Acbay O, SOnsuz A. A controlled trial of gemfibrozil in the treatment of patients with non-alcoholic steatohepaitis. J Hepatol 1999; 31: 384-87.

17. Lavine JE. Vitamin E treatment of non-alcoholic steatohepaitis in children: A pilot study. J Pediatr 2000; 136: 734-38.
18. Marchesini G, Brizi M, Bianchi G, et al. Metformin in non-alcoholic steatohepatitis. Lancet 2001; 358: 893-98.

19. Laurin J, LindonKD,Crippin JS, et al. Urodeoxecholic acid or clofibrate in the treatment of non-alcoholic induced steatohepatitis: a pilot study. Hepatology 1996; 23: 1464-67.

20. Hasegawa T, Yoneda M, Nakamura K, Makino T, Terano A. Plasma transforming growth factor â 1 level and efficacy of á-tocopherol in patients with non-alcoholic steaohepatitis. Alment Pharmacol Ther 2001; 15: 1667-72.

21. Caldwell SH, Hespenhiede EE, Redick JA, Jezzoni JC, Battle EH, Sheppard BL. A pilot study of thiazolidinedione, troglitazone, in non-alcoholic steatohepatitis. Am J Gastroenterol 2001; 96: 51925.

22. Renata B, Stephen A, Harrison. A placebo controlled trial of pioglitazone in subjects with non-alcoholic steatohepatitis. N Eng J Med 2006; 355: 2297-2307.

23. Schaffner F, Thaler H. Non-alcoholic fatty liver disease. Prog Liver Dis 1986; 8: 283-98. 